\title{
Monte Carlo study of phase transition in relativistic heavy ion collisions*
}

\author{
Yu Meiling, Xu Mingmei ${ }^{\dagger}$, Liu Lianshou \\ Institute of Particle Physics, Huazhong Normal University, Wuhan 430079, China \\ E-mail: yumleiopp.ccnu.edu.cn, xummeiopp.ccnu.edu.cn, \\ liulsdiopp.ccnu.edu.cn
}

The hadronization scheme for parton transport in relativistic heavy ion collisions is studied by using Monte Carlo simulation. A simple model is proposed, which has a collective phase transition following a super-cooling, instead of letting the particles to freeze out one by one as done in the traditional event generators. It turns out that the modified model with a sudden phase transition is able to reproduce the experimental longitudinal distributions of final state particles better than the one without phase transition does. The possibility of implementing a transition between different phases other than of the first order is discussed.

Critical Point and Onset of Deconfinement - 4th International Workshop

July 9 - 13, 2007

Darmstadt, Germany

${ }^{*}$ Supported in part by the NSFC-RFBR cooperative research project 10475030 and 05-02-39028-NNSF-a and the NSFC projects $90503001,10775056$.

†peaker. 


\section{Introduction}

There are various phases in QCD. One of them is the confined hadron phase, one is the deconfined quark-gluon-plasma (QGP). The two phases are closely related to the two states of vacuum - the physical vacuum and the perturbative vacuum. Physical vacuum excludes color (or hates color), only colorless hadrons can move in physical vacuum. While the perturbative vacuum admits color degree of freedom, and quarks and gluons can move freely in it. At high temperature and/or high baryon density, the transition from physical vacuum to perturbative vacuum can take place and quarks and gluons can move freely in a volume much larger than hadron size, and thus deconfined quark-gluon-plasma is formed.

Lattice QCD predicts that, in the region with high baryon density and low temperature the transition between normal hadron matter and QGP is a first order phase transition. As the increase of temperature and decrease of baryon density, the line of first order phase transition ends at the critical point and in higher temperature and lower density region, there is a cross-over from normal hadron to QGP.

Up to now, the quark and gluon degree of freedom has been observed in heavy ion collisions, but the nature of the transition is less clear. How to describe the phase transition dynamics microscopically is still a problem.

Transport models [1, 2, 3] are usually used in the microscopic simulation of heavy ion collisions. It simulates the evolution of the particles in detail through considering their interactions elastic and/or inelastic scattering or cascade.

For hadron transport, this kind of models allow the cascade to continue until the interactions stop and then the hadrons freeze-out from the system. A noticeable property of such models is that, each hadron has its own freeze-out time and the hadrons freeze-out one by one.

Such an approach is acceptable for hadron transport. When hadrons stop interacting with each other, they will freeze-out, fly away from the system, and move towards the detectors freely. However, using the same transport picture to describe parton, then partons will also freeze-out oneby-one, and the hadronization is parton-wise. There will be only parton-wise hadronization, no collective phase transition.

It is not a picture for cross over either. Let us first consider the cross over in an electro-magnetic plasma. Suppose we have a neutral atom gas. When temperature raises, atoms will be ionized one by one and eventually cross over to EM plasma. However, this picture could not be simply extended to QCD, because of vacuum problem. If there is a hadron matter consisting of hadrons in physical vacuum, these hadrons decomposed to quarks one by one is impossible, because of the color confinement property of QCD, i.e. isolated color objects can not exist in physical vacuum[4].

Since the above mentioned hadronization scheme in transport model is neither a 1st order phase transition nor a cross over, we need to consider another approach to realize the transition.

In this talk we will concentrate on the 1-st order phase transition, where there is a sudden vacuum change accompanied by collective hadronization. In section 2 we will demonstrate a simple way to add a 1st order phase transition in transport model. We take a presently available model AMPT with string melting - as an example to add a collective phase transition. Our procedure includes 3 steps. a) At first we will look at the time evolution of parton and hadron numbers. We will find that a few partons have unreasonable long lifetime. It's unphysical for partons to propagate in 
physical vacuum. b) So we extract parton temperature by fitting the transverse mass distribution, c) choose an appropriate temperature to add a sudden phase transition with supercooling, we will then truncate the parton transport and let all the left partons be transformed into hadrons. In section 3 is shown the effect of a sudden phase transition to final state.

\section{1st order phase transition in transport model}

\subsection{Brief introduction to AMPT}

AMPT[3] is a multiphase transport model, which is based on non-equilibrium transport dynamics.

There are 4 components of AMPT with string melting (v2.11): the initial conditions, parton transport, hadronization and hadron transport. The initial conditions are generated by HIJING, which generate $(x, p)$ distributions of minijet partons from hard process and strings from soft process. Strings are then melted to partons. After the primary interactions, the time evolution of partons is then treated according to the ZPC model[5]. At present this model includes only twobody elastic scattering with cross section

$$
\sigma_{p} \simeq \frac{9 \pi \alpha_{s}^{2}}{2 \mu^{2}}
$$

Two partons will undergo a scattering when the distance between them is less than $\sqrt{\sigma / \pi}$. After partons stop interactions, a simple quark coalescence model is used to combine the two nearest partons into a meson and three nearest quarks (antiquarks) into a baryon (antibaryon). Scattering among the resulting hadrons are described by ART model[6] which includes baryon-baryon, baryon-meson and meson-meson elastic and inelastic scattering.

It turns out that AMPT with string melting is successful in elliptic flow and two-pion correlation function [7, 8], but failed in describing hadron rapidity and transverse momentum spectra.

In the following we will use AMPT with string melting to generate events of AuAu collisions at $\sqrt{s_{N N}}=200 \mathrm{GeV}$ with impact parameter $b \leq 3 \mathrm{fm}$, parton cross section $10 \mathrm{mb}$.

\subsection{Time evolution of parton and hadron numbers in AMPT}

The ordinary time evolution of parton and hadron yield is easy to get. Each initial parton has a formation time $t_{f}=E_{H} / m_{T, H}^{2}$ with $E_{H}, m_{T, H}$ the energy and transverse mass of its parent hadron. After this formation time, partons start to scatter with each other. When parton interaction ceases, hadrons are produced one by one. Fig. 1 shows the percentage of parton and hadron respectively, at different time after the collision. The percentage at time $t$ is defined as the parton (hadron) numbers at that time divided by the total parton (hadron) numbers appearing in this event. It can be seen that, when $\mathrm{t}<5 \mathrm{fm} / c$, partons dominate, the system as a whole is in the deconfined phase, located in the perturbative vacuum, with a few hadrons evaporated out. As the time increases, the number of partons decreases and the number of hadrons increases. When $t>30 \mathrm{fm} / c$, hadrons dominate, the system is in confined phase, located in physical vacuum. In this hadronization process a parton is transformed to hadron when and only when it ceases to interact with other partons. Thus a few partons can survive up to a long time, e.g. $t \sim 80 \mathrm{fm} / c$. 


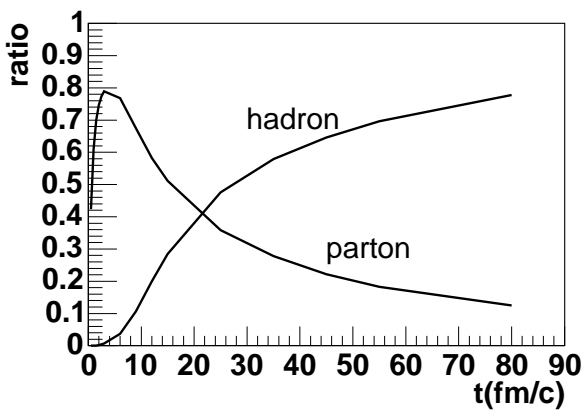

Figure 1: The percentage of partons and hadrons, respectively, in AMPT v2.11 for $\sqrt{s_{N N}}=200 \mathrm{GeV}$ with $b \leq 3 \mathrm{fm}$ and parton cross section $10 \mathrm{mb}$.
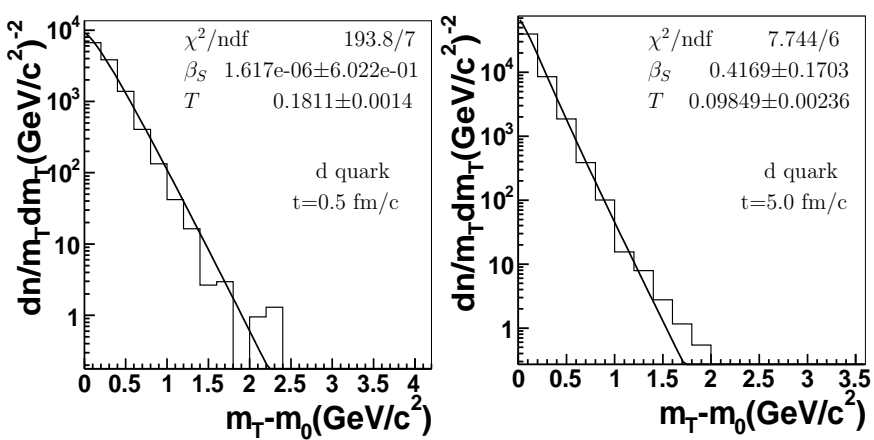

Figure 2: The transverse mass distribution (histograms) for dquarks at two different time $t=0.5 \mathrm{fm} / c$ and $5 \mathrm{fm} / c$. The lines are the fit to Eq.(2.2).

The problem is: there is no collective phase transition but parton-wise hadronization; partons are allowed to propagate in physical vacuum, this is unphysical. A few partons have unreasonable long lifetime, which is inconsistent with the general belief that the existing time of QGP is about $1-5 \mathrm{fm} / c[9]$, and that hadrons freeze out at about $20-40 \mathrm{fm} / c[10]$. Such partons will have infinite energy according to QCD.

To solve the problem, we add a collective phase transition to transport model, truncate the parton evolution.

In principle, the collective phase transition should proceed part by part, and take a certain period of time. There is coexistence of both phases (coexistence of two vacua) in 1st order phase transition. As a first step, we assume that the phase transition takes place immediately in the whole system.

\subsection{Extract temperature from parton and hadron $m_{t}$ distributions}

To study the phase transition, we need to know the evolution of parton temperature. We assume the system is locally thermal equilibrated. Under this assumption a thermal + transverse radial flow model[11] is used to extract the temperature of the system at different time. In this model, the transverse mass distribution is given by

$$
\frac{\mathrm{d} n}{m_{T} \mathrm{~d} m_{T}} \propto m_{T} \int_{0}^{\infty} r \mathrm{~d} r K_{1}\left(\frac{m_{T} \cosh \rho}{T}\right) I_{0}\left(\frac{p_{T} \sinh \rho}{T}\right) .
$$

Here the parameter $\mathrm{T}$ is temperature. In this subsection, we will use this formula to fit $m_{t}$ of parton system at different time and get the parton temperature at each time.

Fig. 2 is a show of a fitting of the transverse mass of d quark at $0.5 \mathrm{fm} / c$ and $5 \mathrm{fm} / c$. When $\mathrm{t}=0.5 \mathrm{fm} / c$ the temperature of the parton system is about $181 \mathrm{MeV}$, slightly above the predicted critical temperature. When $\mathrm{t}=5 \mathrm{fm} / c$, the temperature has already lowered down to $98 \mathrm{MeV}$. That means, in the first few $\mathrm{fm} / \mathrm{c}$ the parton temperature decreases rapidly and soon becomes lower than the expected phase transition temperature.

\subsection{Add a sudden phase transition with supercooling}

The parton temperature becoming lower than the expected phase transition temperature in 
the first few $\mathrm{fm} / \mathrm{c}$ is regarded as a super-cooling effect, and a 1 st order collective phase transition following a super-cooling stage is added. The physical picture is: high temperature QGP expands and evaporates some hadrons. With the decrease of temperature, the parton system supercools to a certain temperature lower than $T_{\mathrm{c}}$, then a sudden phase transition turns all the left partons to hadrons. The time for adding the phase transition is chosen as a model parameter.

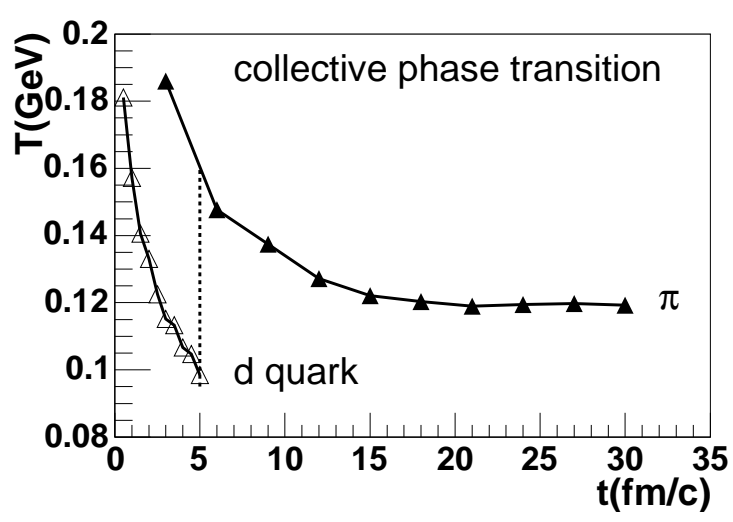

Figure 3: The temperature evolution extracted from parton and hadron transverse mass spectra in AMPT v2.11 with a sudden phase transition implemented at $t=5 \mathrm{fm} / c$.

of expanding.

As such we implement a collective phase transition following a super-cooling to the transport model in a very simple way. We do not intend to construct any satisfactory model at this stage, but only use AMPT as an example to show the importance of collective phase transition.

\section{The effect of collective phase transition on final state}

Let's see the effect of collective phase transition on final state.

First to see the rapidity distribution which is define as $\frac{\mathrm{d} N}{\mathrm{~d} y}=\frac{1}{N_{\mathrm{ev}}} \frac{\Delta n}{\Delta y}$. Fig. 4 shows the rapidity distribution for charged particles, pions, kaons, net protons, protons and antiprotons, respectively. The data in the first pad are from PHOBOS within $6 \%$ centrality with both statistical and systematic errors, and the other data are from BRAHMS within 5\% centrality with only statistical errors. The solid lines represent the result of the AMPT with string melting, the dotted lines are that with a phase transition.

It is clear that dotted lines fit the data better, especially for kaons and protons. That means with phase transition, model describes data better than the original one.

Second to the elliptic flow. The AMPT with string melting, is successful in describing the elliptic flow through adjusting the parton cross section, see the solid lines in Fig. 5. The implementation of phase transition gives almost the same shape, see the dotted lines in Fig. 5, and so preserve the agreement with data. 

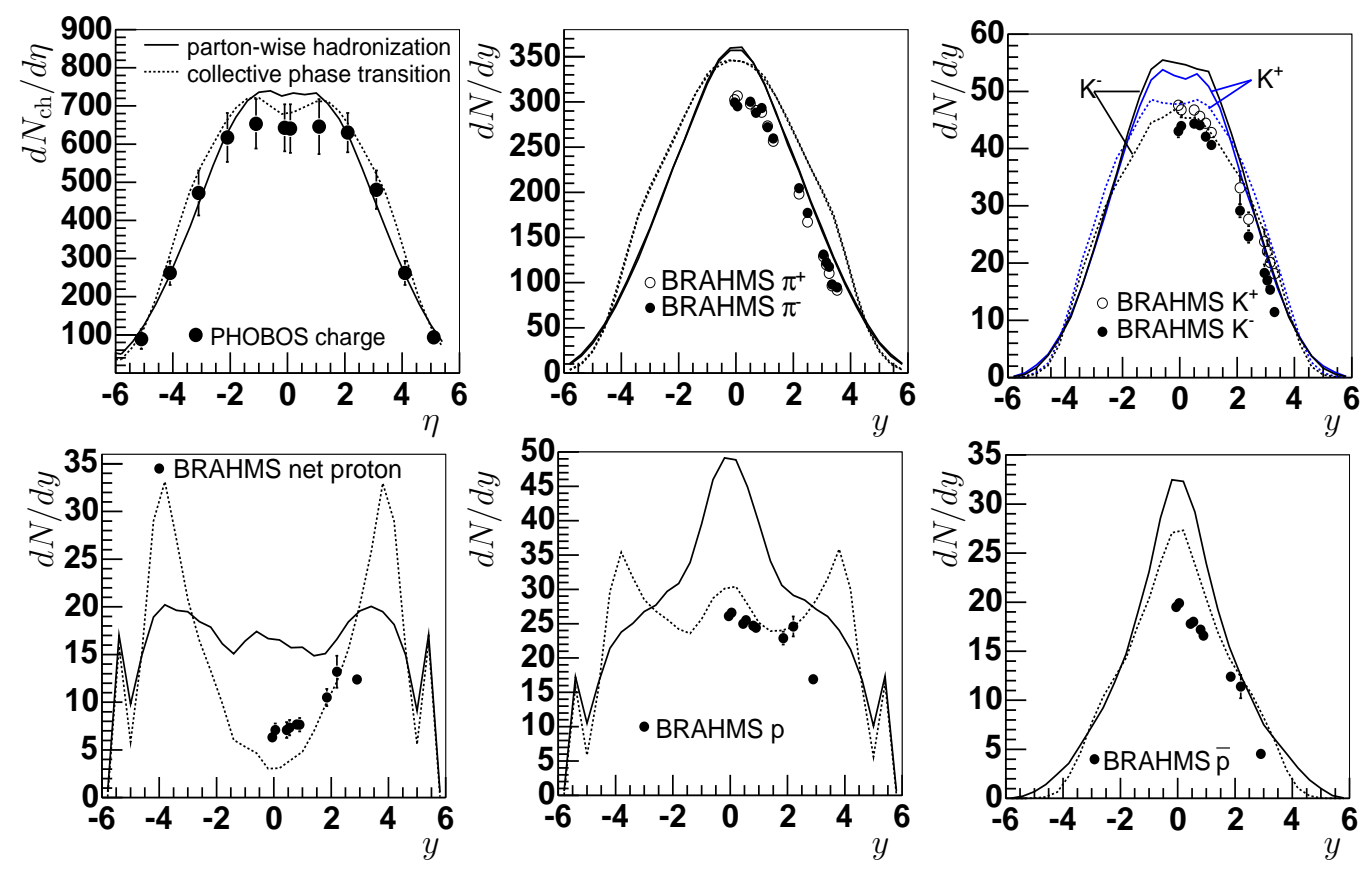

Figure 4: The rapidity distribution for $\sqrt{s_{N N}}=200 \mathrm{GeV}$ Au-Au central collisions. The solid lines are AMPT v2.11 with parton-wise hadronization and the dashed lines are that with sudden phase transition implemented. The impact parameter is $b \leq 3 \mathrm{fm}$ and parton cross section $10 \mathrm{mb}$. The circles are data from PHOBOS 6\% and BRAHMS 5\% central collisions.

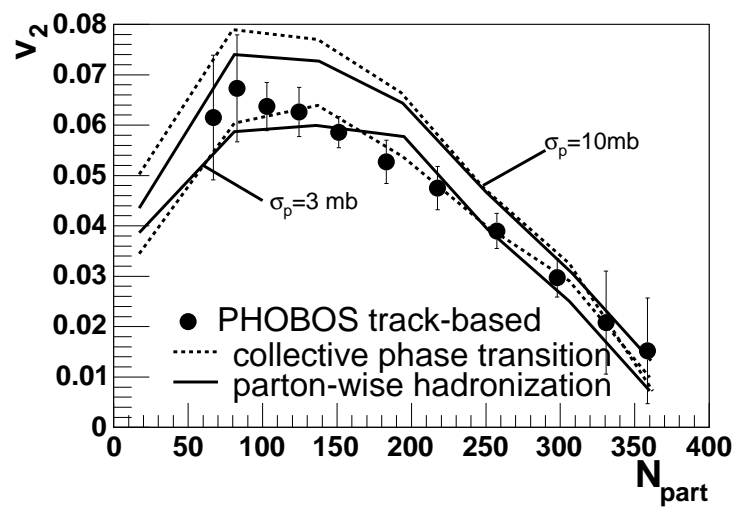

Figure 5: Centrality dependence of charged hadron elliptic flow for $\sqrt{s_{N N}}=200 \mathrm{GeV} \mathrm{Au}-\mathrm{Au}$ central collisions. The solid lines are the AMPT v2.11 with parton-wise hadronization and dashed lines are that with sudden phase transition implemented. The solid circles are data from PHOBOS experiments[12]. The impact parameter is $b \leq 3 \mathrm{fm}$ and parton cross section is $10 \mathrm{mb}$ and $3 \mathrm{mb}$, respectively.

\section{Conclusion and outlook}

Let us discuss briefly why the model with collective phase transition can describe the experimental data better. Fig. 6 plots the rapidity distribution of partons right before hadronization for two cases. The parton transport is truncated in the model with sudden phase transition, and so there are fewer partons in the mid-rapidity region and the distribution peaks at regions with large absolute values of rapidity. This effect results in lower hadron distribution in the mid-rapidity region 


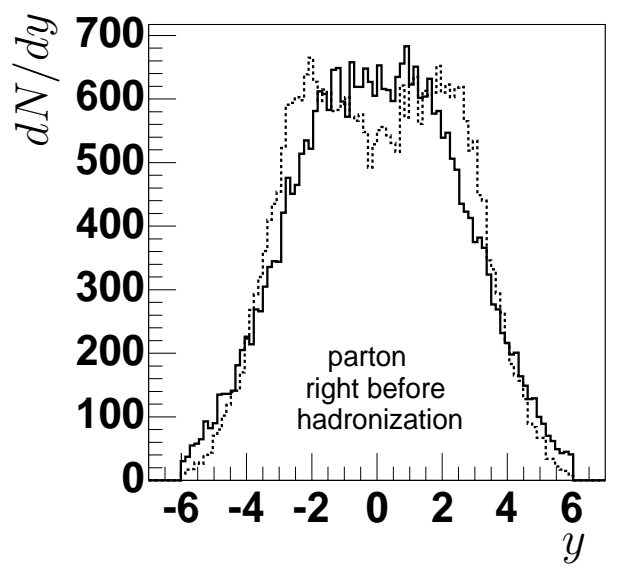

Figure 6: The rapidity distribution of partons right before hadronization in AMPT v2.11 with parton-wise hadronization (solid line)and with collective phase transition (dashed lines).

than that from the original model, which makes the distribution of the final state hadrons closer to the experimental data.

The elliptic flow in parton cascade model is built up very early (less than $5 \mathrm{fm} / \mathrm{c}$ ), thus the truncation of parton transport at $5 \mathrm{fm} / c$ in the present work does not affect the elliptic flow.

In summary, we have proposed a collective phase transition following a super-cooling from parton phase to hadron phase. Though our method for the implementation of phase transition seems to be very crude comparing to the real process, the encouraging results indicate that equilibrium phase transition should be taken into proper account in transport model for relativistic heavy ion collisions.

The microscopic mechanism of phase transition or cross over between normal hadron phase and QGP phase is an interesting topic and still an open question. It is worth thinking over how does the system undergo a phase transition and cross over, it is also worth developing a unified model for both 1 st order phase transition and the cross over and fix the critical point ultimately.

\section{References}

[1] H. Sorge, Phys. Rev. C 52 (1995) 3291.

[2] S.A. Bass et al., Prog. Part. Nucl. Phys. 41 (1998) 225 [nucl-th/9803035].

[3] Z.W. Lin, C.M. Ko, B.A. Li, B. Zhang, and S. Pal, Phys. Rev. C 72 (2005) 064901; Z.W. Lin, S. Pal, C.M. Ko, B.A. Li, and B. Zhang, ibid. 64 (2001) 011902(R) ; B. Zhang, C.M. Ko, B.A. Li, and Z.W. Lin, ibid. 61 (2000) 067901.

[4] See e.g. T.D. Lee, Particle physics and introduction to field theory, Harwood academic publishers, Chur, London, Newyork, 1981, p.400-401.

[5] B. Zhang, Comput. Phys. Commun. 109 (1998) 193.

[6] B.A. Li and C.M. Ko, Phys. Rev. C 52 (1995) 2037.

[7] Z.W. Lin and C.M Ko, Phys. Rev. C 65 (2002) 034904.

[8] Z.W. Lin, C.M. Ko and S. Pal, Phys. Rev. Lett. 89 (2002) 152301. 
[9] T.S. Biro, E. van Doorn, B. Muller, M.H. Thoma, and X.N. Wang, Phys. Rev. C 48 (1993) 1275.

[10] L.P. Csernai and J.I. Kapusta, Phys. Rev. D 46 (1992) 1379; Phys. Rev. Lett. 69 (1992) 737.

[11] E. Schnedermann, J. Sollfrank, and U. Heinz, Phys. Rev. C 48 (1993) 2462.

[12] B.B. Back et al. (PHOBOS Collab.), Phys. Rev. C 72 (2005) 051901(R). 